\title{
Matching Dominating Sets of Interval Graphs
}

\author{
K.Dhanalakshmi \\ Dept. of Applied Mathematics, S.P.Women's \\ University, Tirupati-517502, Andhra Pradesh, India
}

\author{
B.Maheswari \\ Dept. of Applied Mathematics, S.P.Women's \\ University, Tirupati-517502, Andhra Pradesh, India
}

\begin{abstract}
Interval graphs have drawn the attention of many researchers for over 30 years. They are extensively been studied and revealed their practical relevance for modeling problems arising in the real world. The theory of domination in graphs is an enriching area of research at present. In this paper we discuss matching domination number of interval graphs and propose an algorithm for finding matching dominating sets in interval graphs.
\end{abstract}

\section{Keywords}

Interval graph, dominating set, matching dominating set and neighbourhood set

\section{Subject Classification: 68R10}

\section{INTRODUCTION}

The theory of domination in graphs was introduced by Ore [1] and Berge [2] and it has become an emerging area of research in graph theory today. A survey on results and applications of dominating sets was presented by E.J.Cockyane and S.T.Hedetniemi [3].

Let $G(V, E)$ be a graph. A subset $D$ of $V$ is said to be a dominating set of $\mathrm{G}$ if every vertex in $V \backslash D$ is adjacent to a vertex in $D$. The domination number $\gamma(G)$ of $G$ is the minimum cardinality of a dominating set. A matching in a graph $G$ is a subset $M$ of edges of $E$ such that no two edges in $M$ are adjacent. A matching $M$ in $G$ is called a perfect matching if every vertex of $G$ is incident to some edge in $M$.

A dominating set $D$ of $G$ is said to be a matching dominating set if the induced subgraph $\langle D>$ admits a perfect matching. The cardinality of the smallest matching dominating set is called matching domination number and is denoted by $\gamma_{\mathrm{m}}$.

\section{INTERVAL GRAPH}

Let $I=\{1,2, \ldots, n\}$ be an interval family where each i in $I$ is an interval on the real line and $i=\left[a_{i}, b_{i}\right]$ for $i=$ $1,2, \ldots n$. Here $a_{i}$ is called the left endpoint and $b_{i}$ is called the right endpoint of $i$. Without loss of generality, we assume that all endpoints of the intervals in $I$ are distinct numbers between 1 and $2 n$. The intervals are labeled in increasing order of their right endpoints. Two intervals $i$ and $j$ are said to intersect each other if they have non-empty intersection.

Let $G(V, E)$ be a graph. $G$ is called an interval graph if there is a one-to-one correspondence between $V$ and $I$ such that two vertices of $G$ are joined by an edge in $E$ if and only if their corresponding intervals in $I$ intersect.

Let $G$ be the interval graph corresponding to the interval family $I$. Let $n b d[i]$ be defined as the set of vertices adjacent to $i$ including $i$. Let $\min (i)$ denote the smallest interval in $n b d[i]$ and $\max (i)$ denote the largest interval in $n b d[i]$. Define Next $(i)=j$ if and only if $b_{i}<a_{j}$ and there does not exist an interval $k$ such that $b_{i}<a_{k}<a_{j}$. If there is no such $\mathrm{j}$, we define next $(i)=$ null.

We proceed in the following manner to find a minimum matching dominating set of an interval graph.

Define $u(i)=\max (\operatorname{Next}(i))$ and mate $(i)=\max (\operatorname{nbd}(i))$. If Next $(i)=$ null, then $u(i)=$ null. Let LI denote the largest interval in $S$. Then the following Algorithm finds a minimum matching dominating set of an interval graph.

\section{ALGORITHM : MMDS - IG}

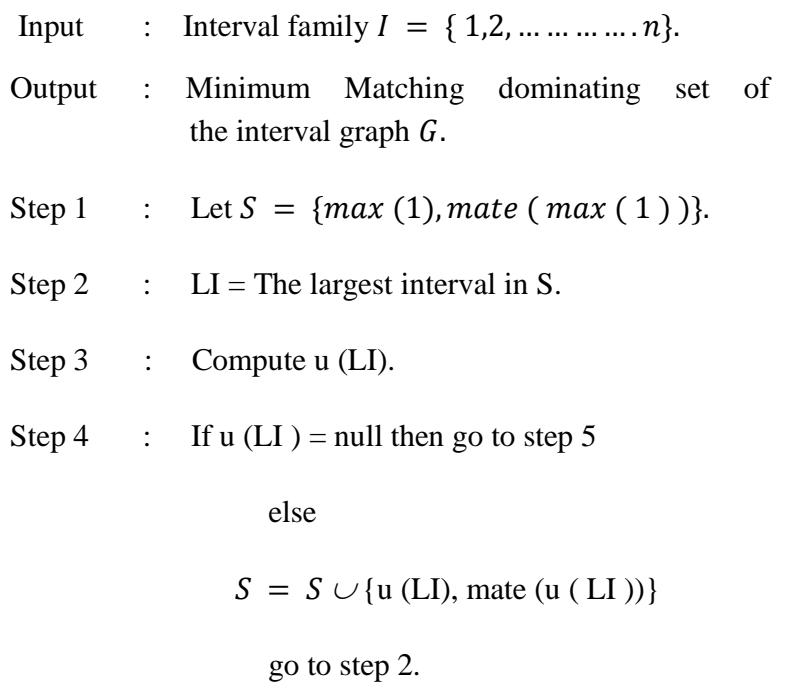

Step $5 \quad: \quad$ End

\section{MAIN RESULTS}

Lemma 1: If $i$ and $k$ are any two intervals which are intersecting and $\mathrm{j}$ is any interval such that $i<j<k$ then $j$ intersects $\mathrm{k}$.

Proof : Since the intervals are labeled in increasing order of their right end points, it is easy to see that when $i<j<k$ then $b_{i}<b_{j}<b_{k}$. Now $i$ intersects $k$ implies that $a_{k}<b_{i}$. Therefore $a_{k}<b_{i}<b_{j}<b_{k}$ which implies that $j$ also intersects $k$.

Lemma 2: If $i$ is any interval and $j$ such that

$i<j<$ mate $(i)$, then $\mathrm{j}$ intersects mate $(i)$.

Proof : By the definition of mate $(i)$, it is the largest interval in nbd $(i)$ and it intersects $i$. Therefore it follows by 
Lemma 1 that, if $j$ is any interval such that $i<j<$ mate $(i)$ then $\mathrm{j}$ intersects mate $(i)$.

Lemma 3 : The intervals between $i$ and mate $(i)$ are dominated by mate $(i)$.

Proof : Follows by Lemma 2.

Lemma 4 : If $i$ is any interval and $j=u(i)=\max (\operatorname{Next}(i))$ then the intervals between $i$ and $j$ are dominated by either $i$ or $j$.

Proof : Let $k$ be an interval between $i$ and $j$.

Case 1: Suppose $i$ does not intersect $k$. Then $b_{i}<a_{k}$. Let $\operatorname{Next}(i)=h$. Then two possibilities arise.

Either $b_{k}>b_{h}$ or $b_{k}<b_{h}$.

Subcase 1 : Suppose $b_{k}<b_{h}$

Then Next (i) $=\mathrm{k}$, so that $h=k$.

Since $j=u(i)=\max (\operatorname{Next}(i))=\max (k)$,

it follows that $k$ interests $j$.

Subcase 2 : Suppose $b_{k}>b_{h}$

Since $j=\max (\operatorname{Next}(\mathrm{i}))=\max (\mathrm{h}), \mathrm{h}$ intersects $j$ and $h<j$.

Now $b_{k}>b_{h}$ implies $h<k$. Therefore $\mathrm{h}$ intersects $j$ and

$h<k<j$ implies by Lemma 1 that $k$ intersects $j$.

Thus in either case $k$ is dominated by $j$.

Case 2: Suppose $k$ does not intersect $j$.

Subcase 1 : Suppose $j$ intersects $i$. Then $a_{j}<b_{i}$. Since $k$ does not intersect $\mathrm{j}$ we have $b_{k}<a_{j}$ so $b_{k}<a_{j}<b_{i}$.

By hypothesis $i<k<j$, so $a_{i}<a_{k}$

Therefore $a_{i}<a_{k}<b_{k}<b_{i}$ and hence $k$ intersects $i$.

Subcase 2 : Suppose $j$ does not intersect $i$. Then $\mathrm{k}$ must intersect $i$, otherwise $k$ becomes the first non - intersecting interval i.e., $\operatorname{Next}(i)=k$. Since $j=u(i)=\max (N \operatorname{ext}(i))=\max (k)$, it follows that $k$ intersects $j$, a contradiction to our assumption. Therefore $k$ must intersect $i$. Thus in either case $k$ is dominated by $i$.

Therefore if $k$ is any interval between $i$ and $j$ where

$j=u(i)=\max (N \operatorname{ext}(i))$ then $k$ is dominated by either $i$ or $j$.

Theorem 1 : The set $S$ produced by the Algorithm is a matching dominating set of the given interval family.

Proof : Let $I$ be the given interval family and $G$ its corresponding interval graph. Let $S$ be the set constructed by the Algorithm

Suppose $S=\left\{i_{1}, i_{2}, \ldots \ldots, i_{k}\right\}$.

Here $i_{1}=\max (1)$ and $i_{2}=$ mate $\left(i_{1}\right)$. So there is an edge between $i_{1}$ and $i_{2}$.Now $i_{3}=\mathrm{u}\left(i_{2}\right)$ and $i_{4}=$ mate $\left(i_{3}\right)$.

Hence there is an edge between $i_{3}$ and $i_{4}$.

Likewise $i_{k}=$ mate $\left(i_{k-1}\right)$ and so there is an edge between $i_{k}$ and $i_{k-1}$. Now $i_{3}=u\left(i_{2}\right)$. So there is no edge between $i_{2}$ and $i_{3}$. Hence there are no edges between $i_{2}$ and $i_{3}$, $i_{4}$ and $i_{5}, \ldots i_{k-2}$ and $i_{k-1}$. By the construction of $S$, it is clear that there are an even number of edges in the induced sub graph $\langle S\rangle$. Therefore $\langle S\rangle$ has a perfect matching.

Hence $S$ is a matching dominating set of given interval graph.

Theorem 2 : The matching dominating set constructed by the Algorithm is minimum.

Proof : Let $S=\left\{i_{1}, i_{2} \ldots \ldots \ldots i_{k}\right\}$ be the matching dominating set constructed by the algorithm.

Let $S_{1}=\left\{j_{1}, . ., j_{m}\right\}$ be a minimum matching dominating set of G. We show that $\left|S_{1}\right|=\left|S_{2}\right|$.

Without loss of generality assume that $j_{1}<j_{2}<. .<j_{m}$. Since the vertices in $S_{1}$ dominate the vertices in $G$ and $S_{1}$ is also minimum, it follows from $i_{1}=\max (1)$ and $i_{2}=$ mate $\left(\mathrm{i}_{1}\right)$ that $\mathrm{j}_{1} \leq \mathrm{i}_{1}, \mathrm{j}_{2} \leq \mathrm{i}_{2}$. Since $i_{3}=u\left(i_{2}\right)$, by a similar argument it follows that $j_{3} \leq i_{3}$ or $\mathrm{i}_{3} \leq \mathrm{j}_{3}$. But $\mathrm{j}_{4} \leq \mathrm{i}_{4}$ and the argument continues like this. That is between every pair of vertices $\left(i_{2 r-1}, i_{2 r}\right)$ of $S$, there is a vertex $j_{2 r}$ from $\mathrm{S}_{1}$ such that $j_{2 r} \leq i_{2 r}$. Since the number of vertices in $S_{1}$ is even, we take $m=21$ for some $l>0$. If $m<k$, then the vertices after $i_{2 l}$ ( here $\mathrm{j}_{\mathrm{m}}=\mathrm{j}_{21} \leq \mathrm{i}_{21}$ ) will not be dominated by any vertex in $\mathrm{S}_{1}$. Therefore we must have $m=k$. Hence the matching dominating set constructed by the algorithm is minimum.

\section{CONCLUSION}

It is interesting to find various graph theoretic concepts of Interval graphs. The authors have studied inverse dominating sets [4], global neighbourhood sets [5], accurate and total accurate dominating sets and bondage numbers of these graphs. Finding matching dominating sets of Interval graphs will enrich the study this concept in Circular-arc graphs and Overlap graphs. An algorithmic approach gives scope and makes easier to characterize these graphs.

\section{ILLUSTRATION}

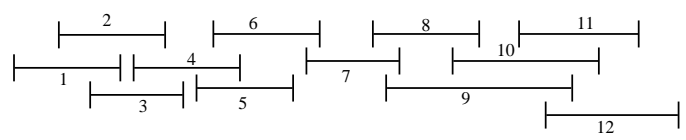

Fig.1

Interval Family I

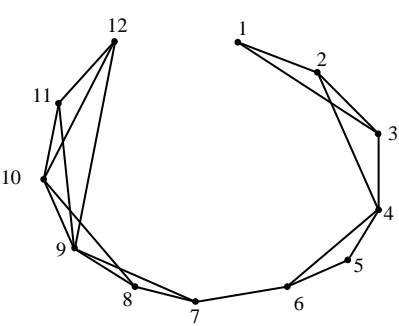

Fig.2

Interval Graph G 


\begin{tabular}{|c|c|c|c|c|c|}
\hline $\operatorname{nbd}(2)$ & $=$ & $\{1,3,4\}$ & $\max (2)=4$ & $\operatorname{Next}(2)=5$ & $u(2)=6$ \\
\hline $\operatorname{nbd}(3)$ & $=$ & $\{1,2,4\}$ & $\max (3)=4$ & $\operatorname{Next}(3)=5$ & $\mathrm{u}(3)=6$ \\
\hline $\operatorname{nbd}(4)$ & $=$ & $\{2,3,5,6\}$ & $\max (4)=6$ & $\operatorname{Next}(4)=7$ & $u(4)=9$ \\
\hline $\operatorname{nbd}(5)$ & $=$ & $\{4,6\}$ & $\max (5)=6$ & $\operatorname{Next}(5)=7$ & $u(5)=9$ \\
\hline $\operatorname{nbd}(6)$ & $=$ & $\{4,5,7\}$ & $\max (6)=7$ & $\operatorname{Next}(6)=8$ & $u(6)=10$ \\
\hline $\operatorname{nbd}(7)$ & $=$ & $\{6,8,9\}$ & $\max (7)=9$ & $\operatorname{Next}(7)=10$ & $\mathrm{u}(7)=12$ \\
\hline $\operatorname{nbd}(8)$ & $=$ & $\{7,9,10\}$ & $\max (8)=10$ & $\operatorname{Next}(8)=11$ & $\mathrm{u}(8)=12$ \\
\hline $\operatorname{nbd}(9)$ & $=$ & $\{7,8,10,11,12\}$ & $\max (9)=12$ & $\operatorname{Next}(9)=$ null & $\mathrm{u}(9)=$ null \\
\hline $\operatorname{nbd}(10)$ & $=$ & $\{8,9,11,12\}$ & $\max (10)=12$ & $\operatorname{Next}(10)=$ null & $\mathrm{u}(10)=$ null \\
\hline $\operatorname{nbd}(11)$ & $=$ & $\{9,10,12\}$ & $\max (11)=12$ & $\operatorname{Next}(11)=$ null & $\mathrm{u}(11)=$ null \\
\hline $\operatorname{nbd}(12)$ & $=$ & $\{9,10,11\}$ & $\max (12)=11$ & $\operatorname{Next}(12)=$ null & $\mathrm{u}(12)=$ null \\
\hline
\end{tabular}

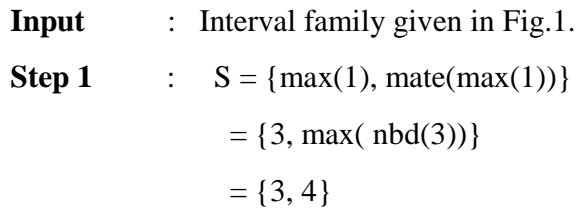

Step 5 : End.

Output : $S=\{3,4,9,12\}$ is the minimum matching dominating set of the interval graph given in Fig. 2.

\section{REFERENCES}

[1] O. Ore, - Theory of Graphs, Amer.Math.Soc.Colloq.Publ.38, Providence (1962).

[2] C. Berge, - Graphs and hypergraphs, North-Holland, Amsterdam(1973).

[3] E.J. Cockayne, and S.T. Hedetniemi, -Towards a theory of domination in graphs, Networks 7,(1977), 247-261.

[4] K.Dhana Lakshmi and B.Maheswari, - Inverse dominating set of Interval graphs - Journal of Current Sciences- Vol.15 no.1 (2010),189-192.

[5] K.Dhana Lakshmi and B.Maheswari, -Minimum Global neighbourhood set of Interval graphs - Journal of Pure and Applied Physics Vol.20,no.3 (2008), 183-187. 